\title{
ANCA-Associated Vasculitis Clinical Presentation and Clinical Predictors of Relapse in Saudi Arabia
}

\author{
Hana Alahmari (iD \\ Hana Al Daajani ${ }^{2}$ \\ Fatimah Alsayed ${ }^{3}$ \\ Abdulrhaman Alrashid ${ }^{4}$ \\ 'Rheumatology Unit, Department of \\ Medicine, King Khalid University \\ Hospital, Abha, Saudi Arabia; \\ ${ }^{2}$ Rheumatology Unit, Department of \\ Medicine, King Fahad Military Medical \\ City, Dammam, Saudi Arabia; \\ ${ }^{3}$ Department of Medicine, King Abdulaziz \\ Medical City, Riyadh, Saudi Arabia; \\ ${ }^{4}$ Rheumatology Unit, Department of \\ Medicine, King Abdulaziz Medical City, \\ Riyadh, Saudi Arabia
}

Background: Anti-neutrophil cytoplasm antibodies-associated vasculitis (AAV) is a rare autoimmune condition with high-relapsing rate and incidence of complications, resulting in increased morbidity and mortality. Characters of patients with anti-neutrophil cytoplasm antibodies-associated vasculitis in Saudi Arabia require further exploration.

Objective: To evaluate the clinical profile, relapse rate and disease-related complications among patients with AAV at a tertiary hospital in Saudi Arabia. To estimate the role of BVAS score at the time of presentation in predicting relapse during the disease course.

Design and Setting: This retrospective cohort study was performed through data collection from patients' records who had AAV, who visited the rheumatology clinic. The collected data involved the demographics of patients and their investigations, medications, and outcomes of treatment. Statistical analysis was executed through SPSS version 26.

Results: Fifty-two patients were eligible for inclusion, while 48 patients were analyzed because of missing data. Females represented $60.4 \%$. Half of the patients were more than 50 years old, and $68.8 \%$ had comorbidities. As for diagnosis, $62.5 \%$ had granulomatosis with polyangiitis, $25 \%$ had eosinophilic granulomatosis with polyangiitis, and $12.5 \%$ had microscopic polyangiitis. The rate of relapse was $31.3 \%$, while the remission rate was $68.8 \%$. Additionally, $66.7 \%$ had lower respiratory involvement, and $43.8 \%$ had renal involvement. More than half of the patients had BVAS score below 14.5 points. The study did not explore a positive correlation between the disease relapse and high BVAS at the first presentation.

Conclusion: Early prediction of relapse and such intervention is of paramount importance in order to avoid accrual of organ damage with treatments that prevent further relapses. BVAS score was not found to be a potential predictor in our study. Future studies are highly endorsed, with prospective design and large sample size to achieve statistical significance for the incidence of relapses and complications.

Keywords: ANCA-associated vasculitis, relapse predictors, BVAS, low complements, rheumatoid factor, relapse criteria

\section{Introduction}

The antineutrophil cytoplasmic antibody (ANCA)-associated vasculitides (AAV) are a collection of relatively rare autoimmune diseases of unknown cause, characterized by inflammatory cell infiltration causing necrosis of blood vessels targeted the medium and small size vessels. ${ }^{1}$ According to The Chapel Hill Consensus Conference definition (CHCC), the AAV comprise granulomatosis with polyangiitis (GPA, formerly known as Wegener's granulomatosis), microscopic polyangiitis (MPA) and eosinophilic granulomatosis with polyangiitis (EGPA, formerly known as Churg-Strauss syndrome). ${ }^{2}$ Antibody against Protease 3 (Anti-PR3) is found in
Correspondence: Hana Alahmari Rheumatology Unit, Department of Medicine, King Khalid University Hospital, P.O Box 64I, Abha, Saudi Arabia Tel +96672417578

Fax+96674I8III

Email Drhana.s@hotmail.com 
$90 \%$ of GPA phenotype, whereas antibody against Myeloperoxidase (anti-MPO) is predominantly detected in MPA and EGPA phenotypes (80\% and 50\%, respectively,). The ANCA seen in the AAVS are predominantly IgG antibodies directed against components of neutrophilic lysosomes or granules. Indirect immunofluorescence of neutrophils demonstrates a cytoplasmic (c-ANCA) or perinuclear ( $\mathrm{p}-\mathrm{ANCA}$ ) pattern of staining; the former correlates with proteinase-3 reactivity (PR3), whilst the latter correlates with reactivity towards myeloperoxidase (MPO). PR3-ANCA is associated predominantly with GPA, whereas MPO-ANCA is seen mainly in patients with MPA or EGPA. ${ }^{3}$ AAV has a fluctuating nature of the disease course with a high percentage of relapse or many relapses.

Mortality rate is extremely fatal. It is about $90 \%$ without treatment especially with renal or pulmonary involvement. Even with treatment, multiple cohort studies and long-term follow-up of international trials have demonstrated relapse rates that vary between $21 \%$ and $89 \%$ at 5 years, depending on the induction and maintenance regimens that were used. ${ }^{4-11}$

Over long-term monitoring of AAV patients, the most prevalent complications are the renal dysfunction, in addition to severe infections, due to the long-term use of corticosteroids. $^{12}$

Accordingly, early detection of relapses is crucial to reduce morbidity and mortality. Many studies explored the perception factors that can predict the disease relapse. Several clinical and immunological factors confer a higher risk of relapse, including a diagnosis of GPA, and/or PR3-ANCA serology at presentation, ANCA positive after remission induction, or a subsequent increase in ANCA titre, ENT involvement, or better renal function (creatinine $<200 \mu \mathrm{mol} / \mathrm{L}$ ), chronic nasal Staphylococcus aureus carriage, and a history of prior relapse. ${ }^{3,13}$ We hypothesized that the initial BVAS might be a valid independent measure that can estimate the future relapse as only few articles have evaluated the predictive value of BVAS for future relapses with ANCA associated vasculitis. $^{14,15}$ Birmingham Vasculitis Activity Score (BVAS) is a well-established and validated measurement tool for disease activity and extension, as BVAS $=0$ defines disease remission and $\mathrm{BVAS} \geq 1$ defines active disease. ${ }^{16}$ Consequently, the present retrospective analysis would explore the characters of AAV patients, with the correlates for increased relapses and complications, in addition to the ability of BVAS to a marker of impending disease relapse.

\section{Patients and Methods}

\section{Study Design}

We retrospectively reviewed the medical records of 50 patients who visited a rheumatology clinic at the National Guard Hospital in Saudi Arabia. Patients who were included are as follows: patients aging above 14 years old who were diagnosed with AAV based on the Chapel Hill Classification (GPA, MPA, EGPA). All patients completed at least one year of follow-up from the first diagnosis. Two patients were excluded due to insufficient medical records.

\section{Data Collection}

Demographic data included age at the onset of disease, gender, marital status, initial clinical manifestations, disease activity at the time of the diagnosis by BVAS and follow-up duration (defined as the period from diagnosis to the last visit). At each relapse, the BVAS score and the time between the first presentation and each relapse using the BVAS version 3 (BVAS v3). Laboratory variables at the time of diagnosis, and each relapse was collected. Namely, blood count CBC, Renal Function test, urinary red blood cell/ white blood cell count, 24-h urinary protein, Inflammatory markers (ESR, CRP), P-ANCA and C-ANCA, Rheumatoid factor RF, Complements level $(\mathrm{C} 3, \mathrm{C} 4)$. C3, C4 are both analyzed by immunoturbidimetry, ANCA is analyzed by indirect immunofluorescence, anti-PR3/anti-MPO antibodies analyzed by antigenspecific enzyme-linked immunosorbent assay (ELISA) and rheumatoid factor analyzed by the agglutination test. Remission was defined as disappearance of clinical disease activity and stabilization or improvement of the kidney function or BVAS $=0 .{ }^{17}$ Resolution of hematuria was also considered criteria for remission. ${ }^{15}$

Relapse was defined as an increase in serum creatinine level accompanied with GFR decline, new RBC cast above 5 cells, new proteinuria above $500 \mathrm{mg}$ over 24 hours not explained by any other secondary causes, worsening or new extrarenal manifestations attributable to active vasculitis after a time of remission, with or without increase in ANCA titers. ${ }^{12}$ Complications encountered (namely, infection required hospitalization, thrombotic events, malignancy and infertility) and disease outcomes were also recorded.

Medications during the remission induction and maintenance phase were identified. All patients received induction therapy with various combinations of corticosteroids 
and immunosuppressants based on the European Vasculitis Study Group (EUVAS) and the EULAR/ERA-EDTA consensus recommendations. ${ }^{18}$ Namely, intravenous Cyclophosphamide, Rituximab, Azathioprine, Methotrexate, Mycophenolate Mofetil, plasma exchange, and Intravenous Immunoglobulin (IVIG).

\section{Statistical Analysis}

All numerical variables were expressed as means and standard deviations, while categorical variables were displayed as counts and percent. Different correlations between relapses and variables with different features were done through Chi-square testing and multivariable logistic regression at a level of significance $p$-value $<0.05$. Statistical analysis was carried out using SPSS IBM software version 26 , USA.

\section{Ethical Considerations}

All patients' identifiable data was kept in a password protected computer, no patient's data was carried in a portable memory device, only the principal investigator had access to full data, as a retrospective study with no direct implication to the subjects involved, informed consent was not required; consequently, the research team requested a waiver of consent for this study.

The study was approved by King Abdullah International Medical Research Center (KAIMRC). IRB NCBE Registration No: H-01-R-005. It was conducted in accordance with the Declaration of Helsinki.

\section{Results}

Fifty-two patients were eligible for inclusion in this study. However, due to missing data in four patients, only 48 patients were included in the analysis. The characters of the included patients are described below.

\section{Demographic Characters of Patients}

Out of the 48 patients, $60.4 \%$ were females, and the mean age of them is 48 years.

As for marital status, $77.1 \%$ of the patients were married. Demographic information is illustrated in Table 1.

\section{Medical History and Clinical Presentation}

Lower respiratory tract system involvement was the most predominant clinical presentation in about $67 \%$, followed by renal involvement in 50\%. Sinusitis was the least common manifestation. Granulomatosis with polyangiitis (GPA) in $62.5 \%$ found to be the most common diagnosis, followed by $25 \%$ with Eosinophilic Granulomatosis with Polyangiitis and $12.5 \%$ with Microscopic polyangiitis. Using the mean value of BVAS, it was categorized into either less than or equal 14.5 points or above 14.5 points, where $56.3 \%$ of the patients had a score below 14.5, as illustrated in Table 1.

Turning to urine analysis, $29 \%$ had positive WBCs, $45 \%$ had positive RBCs, while one third of the patients had significant proteinuria. Other laboratory investigations were also carried out. These investigations included complete blood count, white blood cell, hemoglobin, platelets, kidney functions, and inflammatory markers. The laboratory investigations showed that the patients are commonly anemia, with significant inflammation and renal affections, as described in Table 1. As for the rheumatology investigations, 39.6\% of the patients had C+ ANCA level, while only $4.2 \%$ had glom antibodies, and $14.6 \%$ had a positive rheumatoid factor. As for complements, 6.3\% of patients had low complement $\mathrm{C} 3$ level, and $2.1 \%$ had low complement C4 level.

Those who underwent further respiratory investigations included HRCT and bronchoscopy. 64.6\% had an abnormal CT chest with non-specific findings including parenchymal opacity, granulomatous formation, pleural effusion and hilar lymphadenopathy. As for bronchoscopy, $14.6 \%$ of the patients had a pulmonary hemorrhage. In regard to medications, around half of the patients had received a dose of steroid equal to $1 \mathrm{mg} / \mathrm{kg} /$ day in the induction remission phase, and $45 \%$ had received pulse steroid which is equal to $1 \mathrm{gm}$ per day for 3 to 5 days. Rituximab and intravenous cyclophosphamide have been used almost similarly. Plasma exchange was applied for 11 patients and 3 patients received IVIG.

\section{Disease Course}

About a third of the included patients suffered from relapses $(31.3 \%)$, while the remission rate was $68.8 \%$. As for relapsing patients, $73.3 \%$ of them had only one relapse, while only one patient had three relapses.

Five complications were identified, including serious infection (requiring hospitalization, dialysis, thrombosis, infertility, and malignancy). The most common complication was an infection, occurring in $33.3 \%$ of patients, while malignancy occurred in $2.1 \%$ of patients, as illustrated in Figure 1.

\section{Factors Influencing Relapses}

To understand the factors correlated to relapses and complications in $\mathrm{AAV}$, chi-square testing at a significance 
Table I Patients Demographic Information

\begin{tabular}{|c|c|c|}
\hline Variable & & Value \\
\hline \multicolumn{3}{|l|}{ Gender } \\
\hline Female & 29 & 60.42 \\
\hline Male & 19 & 39.58 \\
\hline Age $Y$ & & 48.80435 \\
\hline \multicolumn{3}{|l|}{ Marital status } \\
\hline Married & 37 & 82.22 \\
\hline Not married & 8 & 17.78 \\
\hline \multicolumn{3}{|l|}{ Diagnosis } \\
\hline GPA & 30 & 62.50 \\
\hline EGPA & 12 & 25.00 \\
\hline MPA & 6 & 12.50 \\
\hline Disease Duration in months & & $38,925.59$ \\
\hline \multicolumn{3}{|l|}{ BVAS score at DX } \\
\hline 14.5 or less & 27 & 56.3 \\
\hline More than 14.5 & 21 & 43.8 \\
\hline \multicolumn{3}{|l|}{ Comorbidities: } \\
\hline Diabetic & 13 & 29.55 \\
\hline Hypertensive & 18 & 41.86 \\
\hline Dyslipidemia & 9 & 20.45 \\
\hline Hypothyroidism & 6 & 13.95 \\
\hline Malignancy & 4 & 9.30 \\
\hline Chronic kidney disease & 4 & 9.52 \\
\hline Cardiac involvement & 8 & 19.05 \\
\hline Upper respiratory involvement & 18 & 41.86 \\
\hline Lower respiratory involvement & 32 & 76.19 \\
\hline Renal involvement & 21 & 50.00 \\
\hline Joint involvement & 13 & 31.71 \\
\hline Skin involvement & 10 & 24.39 \\
\hline Eye involvement & 9 & 21.95 \\
\hline Sinusitis & 2 & 4.65 \\
\hline CNS involvement & 8 & 19.51 \\
\hline Constitutional symptoms & 8 & 19.51 \\
\hline WBC & & $|2.3| \pm 6.86$ \\
\hline HGB & & $10.4 \pm 2.6$ \\
\hline PLTS & & $539.58 \pm 671.54$ \\
\hline CREAT & & $193.84 \pm 225.13$ \\
\hline ESR & & $72.61 \pm 40.07$ \\
\hline CRP & & $82.29 \pm 68.93$ \\
\hline Anti-PR3/Anti MPO & 33 & 91.67 \\
\hline Anti-PR3 & 19 & 39.6 \\
\hline Anti-MPO & 14 & 29.2 \\
\hline Anti-GBM antibody & 2 & 4.2 \\
\hline \multicolumn{3}{|l|}{ C3 } \\
\hline Normal & 15 & 31.3 \\
\hline low & 3 & 6.3 \\
\hline \multicolumn{3}{|l|}{ C4 } \\
\hline Normal & 17 & 35.4 \\
\hline Low & 1 & 2.1 \\
\hline
\end{tabular}

(Continued)
Table I (Continued).

\begin{tabular}{|c|c|c|}
\hline Variable & & Value \\
\hline \multicolumn{3}{|l|}{ Rheumatoid factor } \\
\hline Positive & 7 & 14.6 \\
\hline Negative & 23 & 47.9 \\
\hline WBC above 5 cells in urine & 14 & 29.2 \\
\hline RBC above 5 cells in urine & 22 & 45.8 \\
\hline \multicolumn{3}{|l|}{ Quantification of 24-hour urine protein } \\
\hline$>500 \mathrm{~mL}$ & 14 & 29. \\
\hline$<500 \mathrm{~mL}$ & 14 & 29.2 \\
\hline $\begin{array}{l}\text { Abnormal Findings in high resolution CT, } \\
\text { HRCT }\end{array}$ & 31 & 64.6 \\
\hline Opacity or consolidation on chest CT & 18 & 37.5 \\
\hline Granuloma/ nodules & 12 & 25 \\
\hline Pleural effusion & 6 & 12.5 \\
\hline Cavitation & 4 & 8.3 \\
\hline Hilar lymphadenopathy & 2 & 4.2 \\
\hline \multicolumn{3}{|l|}{ Bronchoscopy findings } \\
\hline Pulmonary hemorrhage & 7 & 14.6 \\
\hline Eosinophilia> $10 \%$ & 1 & 2.1 \\
\hline \multicolumn{3}{|l|}{ Evidence of Vasculitis by biopsy } \\
\hline Kidney & 11 & 23 \\
\hline Lung & 4 & 8 \\
\hline Skin & 3 & 6 \\
\hline \multicolumn{3}{|l|}{ Disease Relapse } \\
\hline Yes & 15 & 31.25 \\
\hline No & 33 & 68.75 \\
\hline \multicolumn{3}{|l|}{ Number of Relapses } \\
\hline No relapse & 33 & 68.75 \\
\hline One relapse & 11 & 22.92 \\
\hline Two relapses & 2 & 4.17 \\
\hline Three relapses & 1 & 2.08 \\
\hline Four relapses & 1 & 2.08 \\
\hline \multicolumn{3}{|l|}{ Induction Medications } \\
\hline CYC IV & 15 & 34.88 \\
\hline RTX & 14 & 32.56 \\
\hline Pulse GC & 22 & $45.83 \%$ \\
\hline GC I mg/day & 26 & $54.17 \%$ \\
\hline MTX & 5 & $10.42 \%$ \\
\hline MMF & 3 & $6.25 \%$ \\
\hline PLEX & 11 & $22.92 \%$ \\
\hline IVIG & 3 & $6.25 \%$ \\
\hline
\end{tabular}

level, p-value $<0.05$, was used for the BVAS variable as well as autoimmune markers. It has been demonstrated that relapses and the number of relapses did not differ between low and high BVAS groups Tables 2 and 3 


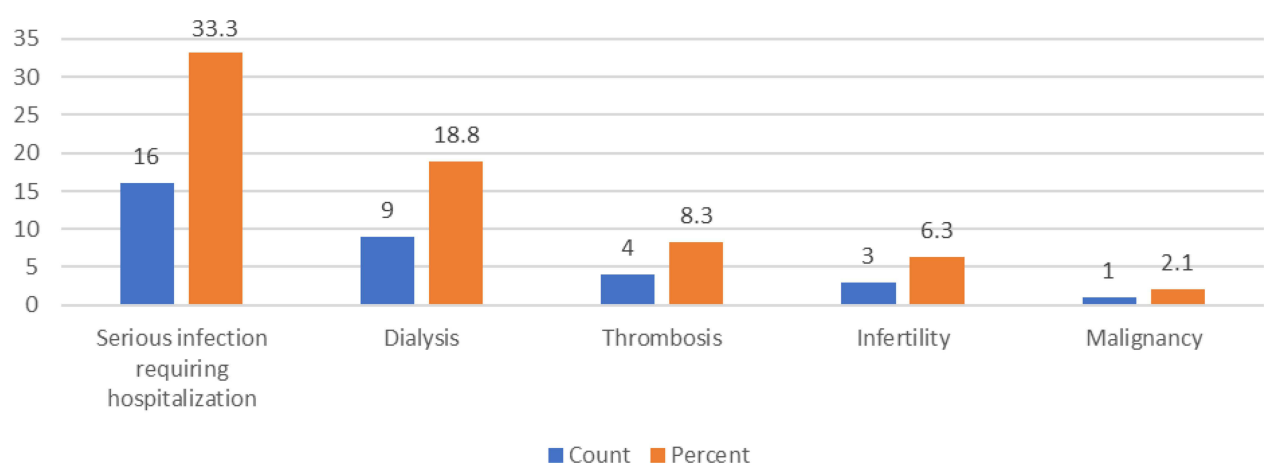

Figure I Disease or therapy related complications.

It has been demonstrated in Table 4 that the incidence of infections did not significantly differ among different doses of cumulative corticosteroids, although this nonsignificant difference was borderline to significant level, which might have been affected by the retrospective nature of the study which may lead to missing or inaccurate data.

\section{Discussion}

Anti-neutrophil cytoplasm antibodies associated with vasculitis are usually associated with clinical manifestations affecting different parts of the body. Though rare, patients with AAV usually have poor prognosis depending on their prognostic factors and severity of the disease. ${ }^{19}$ This prognosis is attributed to the high relapse rate of the disease, in addition to the associated complications, either because of the disease or the administered medications.

The present retrospective investigation assessed the clinical features and characteristics of patients with AAV and their relapse rate and incidence of complications. The study demonstrated that the included patients had a high relapse rate, occurring in almost a third of them. However, threequarters of the relapsing patients had the only relapse. The most diagnosed type of AAV was Granulomatosis with polyangiitis in $62.5 \%$. Additionally, it could be expected that the included patients had a poor prognosis; this is attributed to the high proportion of patients with lower lung involvement (66.7\%) and renal involvement (43.8\%).

Due to the high proportion of patients with lower lung involvement, pulmonary examination revealed that $64.6 \%$ of the patients had an abnormal CT chest scan. Different types of complications were identified, with infection found to be the most commonly occurring complication.

The characters of AAV patients have been explored within varying contexts. A study from Saudi Arabia by Al Arfaj et $\mathrm{al}^{20}$ examined the clinical manifestations and outcomes of ANCA associated AAV in a tertiary center at Riyadh. Al Arfaj et al included 34 patients retrospectively over a duration of 14 years, where $67.6 \%$ had GPA, 26.5\% had EGPA and 5.9\% had MPA. Al Arfaj et al demonstrated that $73.5 \%$ of the AAV patients had pulmonary

Table 2 Correlation Between BVAS at the Time of Diagnosis and Relapses

\begin{tabular}{|c|c|c|c|c|c|}
\hline \multicolumn{6}{|c|}{ 2a. Univariate Logistic Regression Analysis } \\
\hline \multicolumn{2}{|c|}{ Independent Variable } & \multirow[t]{2}{*}{ Odds Ratio } & \multicolumn{2}{|c|}{$95 \% \mathrm{Cl}$ for OR } & \multirow[t]{2}{*}{ P-value } \\
\hline & & & Lower & Upper & \\
\hline \multicolumn{2}{|c|}{$\begin{array}{l}\text { BVAS score at DX } \\
\text { More than } 14.5 \text { vs } 14.5 \text { or less }\end{array}$} & 0.323 & 0.059 & 1.765 & 0.1922 \\
\hline \multicolumn{6}{|c|}{ 2b. Chi-Square Test } \\
\hline & & \multicolumn{3}{|c|}{ BVAS Score } & P-value \\
\hline \multirow[b]{2}{*}{ Relapses } & & \multicolumn{2}{|c|}{ Less than or equal to 14.5} & $\begin{array}{c}\text { More than } \\
\quad 14.5\end{array}$ & \multirow[t]{2}{*}{0.366} \\
\hline & $\begin{array}{l}\text { Yes } \\
\text { No }\end{array}$ & \multicolumn{2}{|c|}{$\begin{array}{l}37.0 \% \\
23.8 \%\end{array}$} & $\begin{array}{l}63.0 \% \\
76.2 \%\end{array}$ & \\
\hline
\end{tabular}


Table 3 Correlation Between Serology Autoimmune Markers Positivity and Disease Relapse

\begin{tabular}{|c|c|c|c|c|c|c|c|}
\hline \multicolumn{8}{|c|}{ 3a. Correlation Between RF and AAV Relapse, Chi-Square Test. } \\
\hline & & & \multicolumn{4}{|c|}{ Rheumatoid Factor } & \multirow{4}{*}{$\begin{array}{c}\text { P-value } \\
0.066\end{array}$} \\
\hline & & & Positive & Negative & & lone & \\
\hline \multirow{2}{*}{\multicolumn{2}{|c|}{ Relapses }} & Yes & $20.0 \%$ & $66.7 \%$ & \multirow{2}{*}{\multicolumn{2}{|c|}{$\begin{array}{l}13.3 \% \\
48.5 \%\end{array}$}} & \\
\hline & & No & $12.1 \%$ & $39.4 \%$ & & & \\
\hline \multicolumn{8}{|c|}{ 3b. Correlation Between ANCA and AAV Relapse, Chi-Square Test. } \\
\hline & & \multicolumn{5}{|c|}{ ANCA Result } & P-value \\
\hline & & Anti-PR3+ & Anti-MPO+ & Both negative & \multicolumn{2}{|c|}{ Not done } & \\
\hline Relapses & Yes & $31.6 \%$ & $50.0 \%$ & $33.3 \%$ & \multirow{2}{*}{\multicolumn{2}{|c|}{$\begin{array}{l}25.0 \% \\
75.0 \%\end{array}$}} & 0.681 \\
\hline & No & $68.4 \%$ & $50.0 \%$ & $66.7 \%$ & & & \\
\hline \multicolumn{8}{|c|}{ 3c. Correlation Between Complements Revels and AAV Relapse, Chi-Square Test. } \\
\hline & & \multicolumn{2}{|c|}{ Complement C3 } & P-value & \multicolumn{2}{|c|}{ Complement C4 } & P-value \\
\hline & & Low & Normal & & Low & Normal & \\
\hline \multirow[t]{2}{*}{ Relapses } & Yes & $33.3 \%$ & $40.0 \%$ & 0.659 & $0.0 \%$ & $41.2 \%$ & 0.466 \\
\hline & No & $66.7 \%$ & $60.0 \%$ & & $100.0 \%$ & $58.8 \%$ & \\
\hline
\end{tabular}

Table 4 Correlation Between Cumulative Steroid Dose Over the First Six Months and Its Relationship with Serious Infections

\begin{tabular}{|l|c|c|c|c|}
\hline \multicolumn{2}{|c|}{} & \multicolumn{2}{|c|}{ Cumulative Steroid Dose } & \multirow{2}{*}{ P-value } \\
\cline { 3 - 4 } \multicolumn{2}{|c|}{} & Dose < $\mathbf{3 0 9 5} \mathbf{~ m g}$ & Dose > $\mathbf{3 0 9 5}$ & \\
\hline \multirow{2}{*}{ Infection } & Yes & $16.7 \%$ & $47.1 \%$ & 0.053 \\
& No & $83.3 \%$ & $52.9 \%$ & \\
\hline
\end{tabular}

involvement. Furthermore, $79.4 \%$ of the AAV patients had ANCA positive, of them $77.8 \%$ had C+ ANCA levels. Al Arfaj et al also reported that the incidence of infections among the included patients was $29.4 \%$. As for treatment, all the patients used corticosteroids, while half of the patients used azathioprine, 58.8\% used cyclophosphamide and $11.8 \%$ used rituximab.

Similar to Al Arfaj et al, the present study showed that GPA was the most common disease type, followed by EGPA and MPA had the least prevalence. Also, pulmonary involvement (particularly lower respiratory involvement) was the most reported in the present study. However, C +ANCA has been lower in the present study compared to Al Arfaj et al, while the incidence of infection was higher. In the present study, renal and lower respiratory involvement were the most commonly seen among the included cohort (66.7\% and $43.8 \%$, respectively). However, $64.6 \%$ had an abnormality in their CT chest findings, and there were urine analysis abnormalities $(29.2 \%$ had positive WBCs, $45.8 \%$ had positive RBCs, while $29.2 \%$ had protein in urine more than $500 \mathrm{~mL}$ over 24 hours) confirming the renal involvement. The relapse rate was $31.3 \%$, although relapses were higher among patients with BVAS score $>14.5 \%$, the difference could not reach statistical significance, which could be attributed to the smaller sample size.

Similarly, Juyoung Yoo et al concluded that the relapse is similar between high BVA, which was 4.7 , and above and below. $^{21}$ Through retrospective screening of 90 patients records and a mean follow up of 42 months, Oh et $\mathrm{al}^{14}$ demonstrated that BVAS score above 13.5 was significantly higher among patients who had relapses. In a large Spanish study by Solans-Laqué et al ${ }^{22}$ that included 550 patients, the predictive ability of BVAS score for future relapses was also demonstrated. Additionally, Solans-Laqué et al demonstrated a significant correlation between BVAS score and mortality, in addition to an increased predictive accuracy for future relapses. However, the fact that different cutoff score defines high BVAS is widely variable. Among these studies, the variability of the definition of high BVAS makes it difficult to draw a conclusion. Furthermore, relapses did not significantly differ based on the rheumatoid factor positivity; only one study in the literature examining the correlation between positive RF and relapse was done by Moon et $\mathrm{al}^{23}$ and it was 
only falsely positive during the follow-up with no prognostic significance.

Although a consistent finding of C-ANCA or PR3 ANCA positive is a significant risk of disease relapse, the present study showed that patients with $\mathrm{P}+$ ANCA results were at higher risk of relapse, but it could not reach statistical significance (Table 3). Similar to Oh et al, ${ }^{14}$ there was a non-significant difference in ANCA results among relapsing and non-relapsing patients.

As for the level of complements, it has been revealed in many studies that low C3 level is negatively correlated with poor prognosis and poor renal survival. ${ }^{24-26}$ there was a nonsignificant difference in the rate of relapses and the number of relapses among patients with low and normal C3 or C4 levels and that was not studied before. Nevertheless, due to the retrospective nature of the present study, it suffered from some limitations. Due to the disease's rarity, small sample size was included, which had affected achieving statistical significance for some correlation. Furthermore, due to the retrospective data collection, some information was missing or incomplete in patients' records. Consequently, future trials should consider these barriers.

\section{Conclusion}

Early prediction of relapse and such intervention is of paramount importance in order to avoid accrual of organ damage with treatments that prevent further relapses. BVAS score is a potential predictor that requires further investigations with larger sample size. Future studies are highly endorsed, with prospective design and large sample size to achieve statistical significance for the incidence of relapses and complications.

\section{Disclosure}

The authors reported no conflicts of interest for this work.

\section{References}

1. Chen Z, Lin L, Yang W, Chen N, Lin Y. Clinical characteristics and prognostic risk factors of anti-neutrophil cytoplasmic antibody (ANCA)-associated vasculitides (AAV). Int Immunopharmacol. 2020;87:106819. doi:10.1016/j.intimp.2020.106819

2. Yates M, Watts R. ANCA-associated vasculitis. Clin Med (Northfield Il). 2017;17(1):60-64. doi:10.7861/clinmedicine.17-1-60

3. Guidelines-for-the-Management-of-ANCA-Associated-Vasculitis-v1. 1[20754].pdf.

4. Jayne D, Rasmussen N, Andrassy K, et al. A randomized trial of maintenance therapy for vasculitis associated with antineutrophil cytoplasmic autoantibodies. $N$ Engl $J$ Med. 2003;349(1):36-44. doi:10.1056/NEJMoa020286

5. De Groot K, Rasmussen N, Bacon PA, et al. Randomized trial of cyclophosphamide versus methotrexate for induction of remission in early systemic antineutrophil cytoplasmic antibody-associated vasculitis. Arthritis Rheumatism. 2005;52(8):2461-2469. doi:10.1002/art.21142
6. de Groot K, Harper L, Jayne DR, et al. Pulse versus daily oral cyclophosphamide for induction of remission in antineutrophil cytoplasmic antibody - associated vasculitis: a randomized trial. Ann Intern Med. 2009;150(10):670-680. doi:10.7326/0003-4819-150-10200905190-00004

7. Pagnoux C, Mahr A, Hamidou MA, et al. Azathioprine or methotrexate maintenance for ANCA-associated vasculitis. $N$ Engl $J$ Med. 2008;359(26):2790-2803. doi:10.1056/NEJMoa0802311

8. Hiemstra TF, Walsh M, Mahr A, et al. Mycophenolate mofetil vs azathioprine for remission maintenance in antineutrophil cytoplasmic antibody-associated vasculitis: a randomized controlled trial. JAMA. 2010;304(21):2381-2388. doi:10.1001/jama.2010.1658

9. Guillevin L, Pagnoux C, Karras A, et al. Rituximab versus azathioprine for maintenance in ANCA-associated vasculitis. $N$ Engl J Med. 2014;371(19):1771-1780.

10. Stone JH, Merkel PA, Spiera R, et al. Rituximab versus cyclophosphamide for ANCA-associated vasculitis. $N$ Engl J Med. 2010;363 (3):221-232. doi:10.1056/NEJMoa0909905

11. Jones RB, Cohen Tervaert JW, Hauser T, et al. Rituximab versus cyclophosphamide in ANCA-associated renal vasculitis. $N$ Engl J Med. 2010;363(3):211-220. doi:10.1056/NEJMoa0909169

12. Pu L, Li GS, Zou YR, Zhang P, Wang L. Clinical predictors of outcome in patients with anti-neutrophil cytoplasmic autoantibody-related renal vasculitis: experiences from a single-center. Chin Med J. 2017;130 (8):899-905. doi:10.4103/0366-6999.204099

13. Walsh M, Flossmann O, Berden A, et al. Risk factors for relapse of antineutrophil cytoplasmic antibody-associated vasculitis. Arthritis Rheum. 2012;64(2):542-548. doi:10.1002/art.33361

14. Oh YJ, Ahn SS, Park ES, et al. Chest and renal involvements, Birmingham vascular activity score more than 13.5 and five factor score (1996) more than 1 at diagnosis are significant predictors of relapse of microscopic polyangiitis. Clin Exp Rheumatol. 2017;35 Suppl 103(1):47-54.

15. Á H, Polner K, Arányi J, et al. Simple, readily available clinical indices predict early and late mortality among patients with ANCA-associated vasculitis. BMC Nephrol. 2017;18(1):76. doi:10.1186/s12882-017-0491-z

16. Stone JH, Hoffman GS, Merkel PA, et al. A disease-specific activity index for wegener's granulomatosis: modification of the Birmingham vasculitis activity score. International Network for the Study of the Systemic Vasculitides (INSSYS). Arthritis Rheum. 2001;44(4):912-920. doi:10.1002/1529-0131(200104)44:4<912::AID-ANR148>3.0.CO;2-5

17. Mukhtyar C, Hellmich B, Jayne D, Flossmann O, Luqmani R. Remission in antineutrophil cytoplasmic antibody-associated systemic vasculitis. Clin Exp Rheumatol. 2006;24:S-93.

18. Wallace ZS, Miloslavsky EM. Management of ANCA associated vasculitis. BMJ. 2020;368:m421.

19. Murosaki T, Sato T, Akiyama Y, Nagatani K, Minota S. Difference in relapse-rate and clinical phenotype by autoantibody-subtype in Japanese patients with anti-neutrophil cytoplasmic antibody-associated vasculitis. Mod Rheumatol. 2017;27(1):95-101. doi:10.1080/14397595.2016.1192760

20. Al Arfaj AS, Khalil N. ANCA associated vasc ulitis in patients from Saudi Arabia. Pak J Med Sci. 2018;34(1):88. doi:10.12669/ pjms.341.13881

21. Yoo J, Kim HJ, Jung SM, Song JJ, Park YB, Lee SW. Birmingham vasculitis activity score of more than 9.5 at diagnosis is an independent predictor of refractory disease in granulomatosis with polyangiitis. Int J Rheum Dis. 2017;20(10):1593-1605. doi:10.1111/ 1756-185X.13144

22. Solans-Laqué R, Rodriguez-Carballeira M, Rios-Blanco JJ, et al. Comparison of the Birmingham vasculitis activity score and the five-factor score to assess survival in antineutrophil cytoplasmic antibody-associated vasculitis: a study of 550 patients from Spain (REVAS Registry). Arthritis Care \& ReS. 2020;72(7):1001-1010. doi:10.1002/acr.23912 
23. Moon JS, Lee DD, Park YB, Lee SW. Rheumatoid factor false positivity in patients with ANCA-associated vasculitis not having medical conditions producing rheumatoid factor. Clin Rheumatol. 2018;37(10):2771-2779. doi:10.1007/s10067-017-3902-4

24. Augusto JF, Langs V, Demiselle J, et al. Low serum complement C3 levels at diagnosis of renal ANCA-associated vasculitis is associated with poor prognosis. PLoS One. 2016;11(7):e0158871. doi:10.1371/ journal.pone. 0158871

25. Choi H, Kim Y, Jung SM, Song JJ, Park YB, Lee SW. Low serum complement 3 level is associated with severe ANCA-associated vasculitis at diagnosis. Clin Exp Nephrol. 2019;23(2):223-230. doi:10.1007/s10157-018-1634-7
26. Deshayes S, Aouba A, Khoy K, Mariotte D, Lobbedez T, Silva N. Hypocomplementemia is associated with worse renal survival in ANCA-positive granulomatosis with polyangiitis and microscopic polyangiitis. PLoS One. 2018;13(4):e0195680. doi:10.1371/journal. pone. 0195680

\section{Publish your work in this journal}

Open Access Rheumatology Research and Reviews is an international, peer-reviewed, open access journal publishing original research, reports, editorials, reviews and commentaries on all aspects of clinical and experimental rheumatology in the clinic and laboratory including the following topics: Pathology, pathophysiology of rheumatological diseases; Investigation, treatment and management of rheumatological diseases; Clinical trials and novel pharmacological approaches for the treatment of rheumatological disorders. The manuscript management system is completely online and includes a very quick and fair peer-review system, which is all easy to use Visit http://www.dovepress.com/testimonials.php to read real quotes from published authors. 